niet minder zijp; in dit opzicht in de vergelijking lust schen de trajekten 9bI en 9blI zeer merkwaardig.

Onclanks het niet-stoppen te Amersfcort was de gemiddelde uursnelheid op het trajekt $4 \mathrm{~b}$ niet meer, maar geringer dan die van de te Amersfoort stoppende ; een bewijs te meer, dat het doorriden op dat station meer het gevolg van de concurrentie (vliegenafvangerij) was dan van tijdsbesparing.

Moge een volgend 10-tal jaren een minder gebroken statistiek brengen, tenzij, dat de invoering van een geregelden luchtvaartadienst een geheele verschuiving te weeg brengt.

Mr. L. G. N. Bouricius.

\title{
Bijlage A.
}

\section{KORTSTE RIJTIJDEN OP ENKELE TRAJECTEN.}

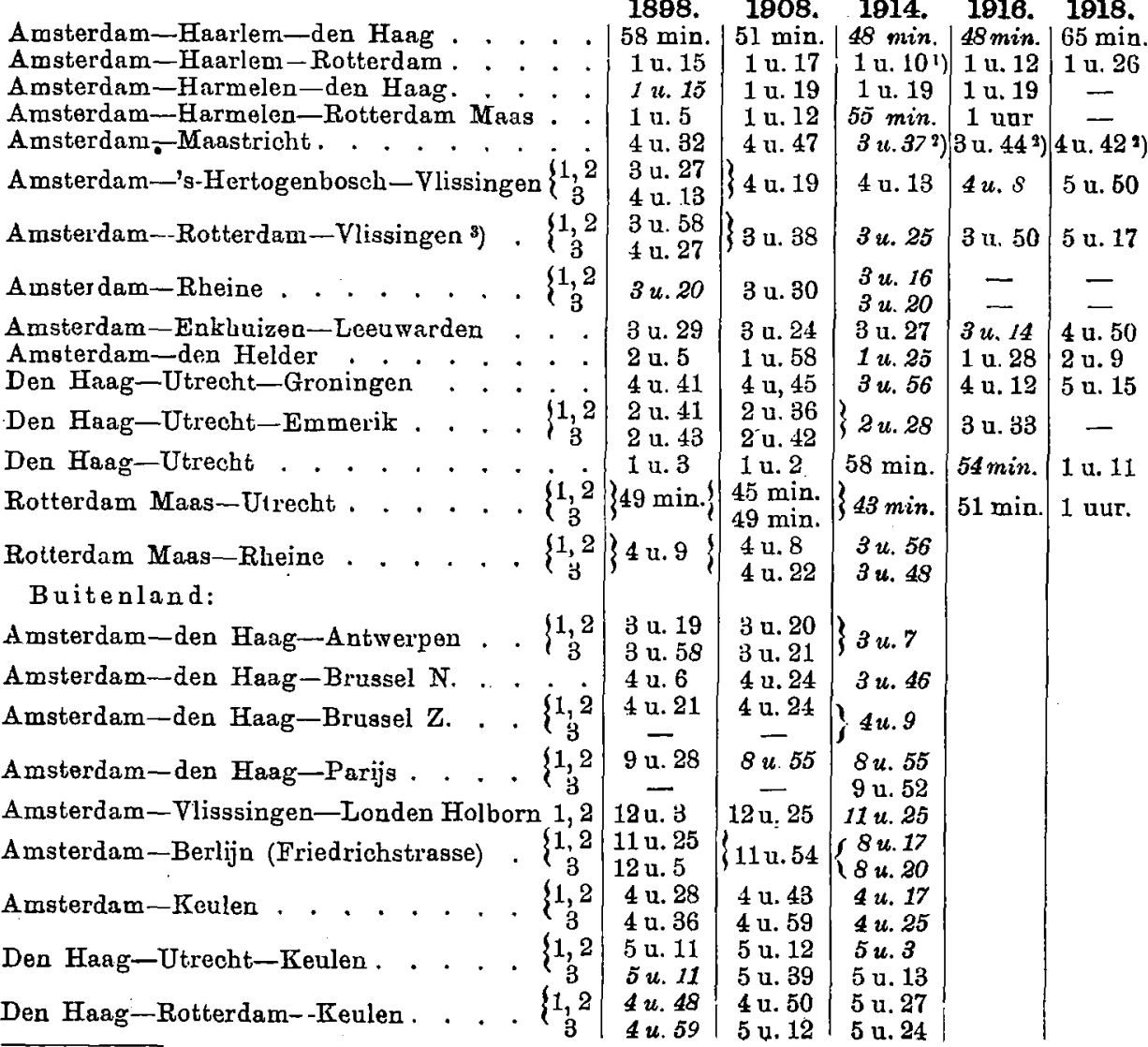

1) Ook in 1888 zonder stoppen in den Haag.

3) Over Weert.

8) 1898,1916 en 1918 over den Haag; 1908 en 1914 over Gouda. 
$\begin{array}{lll}1878 & 1908 & 1914\end{array}$

1. Er had sneltreinverkeer plaats over K.M. of in verhouding tot 1878

2. Sneltreinen met alleen $1 *$ en $2 \cdot$ klasse verkeerden over weg-K.M. . of in verhouding tot $1878^{\circ}{ }^{\circ}{ }^{\circ}{ }^{\circ} \circ$ en in verhouding tot het totaal aantal wegK.M. (1) . . . . . . . . . . . . . \%

3. De $3^{\circ}$ klasse werd in sneltreinen vervoerd over weg-K M. . of in verhouding tot $1878 \dot{\circ}^{\circ} \circ$ en in verbouding tot het totaal antal wegK.M. (1)

4. De 10 klasse ontbrak in sneltreinen op weg-K.M. of in verhouding tot het totaal aantal wegK.M. (1) . . . . . . : . . . . . . .\%

5. De 10 klasse ontbrak in sneltreinen geheel op weg-K.M. of in verhouding tot het totaal antal weg: K.M. (1)

Het antal anelt of in verhouding tot 1878 . $\%$ sneltreinen-K.M. op 1 weg-K.M. (1) . . . . .

7. Van de sneltreinen vervoerden alleen $1^{\circ}$ en $2^{\circ}$ klasse K.M. . . . . . . . . . . . . . . of in verhouding tot 1878 . . . . . . . \% en per weg-K.M. (2) . . . . . . . . . .

8. De $3^{\circ}$ klasse werd gevoerd in sneltrein-K.M. of in verhouding tot 1878 per weg-K.M. en in verhouding tot het totaal aantal sneltreinK.M. (6) . . . . . . . . . . . .\%

9. De 10 klasse ontbrak in sneltrein-K.M. . . . of per weg-K.M. (4) . K.M. $(6)$

Lijn Amsterdam-Rotterdam, lang $87 \boldsymbol{K} . \boldsymbol{M}$.

10. Het aantal sneltrein-K.M. bedroeg of in verhouding tot 1878 en per weg-K.M.

11. Van de sneltreinen voerden alleen $1^{\bullet}$ en $2^{\circ}$ klasse en per weg-K.M.

12. De $3^{\circ}$ klasse werd gevoerd in trein-K $\mathrm{M}$. of in verhouding tot 1878 . . . . . . . \% per weg-K.M.

en in verhouding tot het totaal aantal sneltreinK.M. (10)

\section{Oude Rïnspoorlïnen, lang $K . M$.}

13. Het aantal sneltrein-K.M. bedroeg of in verboud'ng tot 1878 en per weg-K.M.

14. Sneltreinen zonder $3^{\circ}$ klasse reden op veg-K.M. in verhouding tot het geheel . . . . . .

15. Van de sneltreinen voerden alleen $1^{\circ}$ en $2^{\circ} \mathrm{klass}$ trein-K.M. of in verhouding tot 1878 . $\quad . \quad . \quad . \quad . \quad . \quad \%$ en per weg-K.M. (14)......

16. De $3^{e}$ klasse werd gevoerd in sneltrein-K.M. of in verhouding tot 1878 . . . . . . \% per weg-K.M. en in verhouding tot het totaal aental sneltroinK.M. (13) .

\begin{tabular}{|c|c|c|c|c|}
\hline & & & & \\
\hline $\begin{array}{c}1241 \\
00,00\end{array}$ & $\begin{array}{r}2199 \\
177,20\end{array}$ & $\begin{array}{r}2352 \\
189,52\end{array}$ & $\begin{array}{r}.2132 \\
171,79\end{array}$ & $\begin{array}{r}1849 \\
148,27\end{array}$ \\
\hline 0 & 70 & 026 & & \\
\hline 00,0 & & 101,48 & 0,00 & 0,00 \\
\hline 81,47 & 36,61 & 43,62 & 0,00 & 0,00 \\
\hline $\begin{array}{r}797 \\
0,00\end{array}$ & $\begin{array}{r}2175 \\
272,90\end{array}$ & \begin{tabular}{|r|}
2328 \\
292,10
\end{tabular} & $\begin{array}{r}2132 \\
267.50\end{array}$ & $\begin{array}{r}1840 \\
230,87\end{array}$ \\
\hline 64,22 & $\begin{array}{r}3,91 \\
36\end{array}$ & $\begin{array}{r}98,98 \\
111\end{array}$ & $\begin{array}{r}0,00 \\
121\end{array}$ & $\begin{array}{r}00,00 \\
35\end{array}$ \\
\hline- & 1,64 & 4,72 & 5,68 & 1,90 \\
\hline - & - & 24 & 67 & 3 \\
\hline & - & 1,00 & 3,14 & 1,90 \\
\hline 7811 & 31664 & 45650 & 98 & 1295 \\
\hline 0,00 & 405,38 & 584,43 & 464,70 & 165,83 \\
\hline 6,29 & 14,40 & 19,41 & 17,03 & 7,06 \\
\hline 3709 & 2795 & 269 & & - \\
\hline 100 & & & 0,00 & 0,00 \\
\hline & & & 0 & 0.00 \\
\hline 4. & 288 & 42381 & & 12953 \\
\hline 0.00 & 703,78 & 1038,18 & 39 & 315,77 \\
\hline 5,15 & 13,28 & 18,20 & 2 & 7,05 \\
\hline 52,52 &, 17 & 2,84 & & 100,00 \\
\hline & 68 & & & 123 \\
\hline- & 8 & 35,02 & 31,56 & 35,2 \\
\hline - & 0,91 & 8,51 & 10.52 & 9,0 \\
\hline 992 & & 76 & & 23 \\
\hline, 00 & & & & 217.58 \\
\hline 55 & , 6 & ,69 & 66,30 & 27,3 \\
\hline 522 & & & 一 & - \\
\hline 0,00 & & & 0,00 & 0,00 \\
\hline & & & - & - \\
\hline 570 & 4 & 4 & & 2376 \\
\hline 0,00 & & & & 416.84 \\
\hline$f_{5}$ & 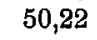 & 66,3 & 66. & 78 \\
\hline 52,20 & 75 & 90,02 & 100,00 & 100,0 \\
\hline 212 & & 12 & 202 & 18 \\
\hline 15 & 8432 & 10264 & 6808 & 2156 \\
\hline & & & & \\
\hline & & & 33,70 & 11,9 \\
\hline & & & & \\
\hline 0 &, 75 & 75 & 00 & $0,0 c$ \\
\hline & & & - & $\ldots$ \\
\hline & & & 00 & 0 \\
\hline & & & & 1 \\
\hline & & & & 61 \\
\hline 00,00 & 38 & 485,28 & 336,37 & 100,5 \\
\hline & 37,14 & 46,33 & 0 & 11,9 \\
\hline & & 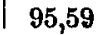 & 100,00 & 10 \\
\hline
\end{tabular}


Bijlage C.

Afstanden van meer dan $^{*} 40$ K.M

\begin{tabular}{|c|c|c|c|c|c|c|c|c|c|c|}
\hline \multirow{3}{*}{$\mathrm{N}^{\circ}$. } & \multirow{3}{*}{ Traject. } & \multirow{3}{*}{ K.M. } & \multicolumn{4}{|c|}{1908} & \multicolumn{4}{|c|}{1898} \\
\hline & & & \multirow{2}{*}{$\begin{array}{l}\text { Aantal } \\
\text { troinell. }\end{array}$} & \multicolumn{2}{|c|}{ Kortste duur } & \multirow{2}{*}{$\begin{array}{c}\mathrm{KM} \text {. } \\
\text { per } \\
\text { uur. }\end{array}$} & \multirow{2}{*}{$\begin{array}{l}\text { Aa tal } \\
\text { tecinon. }\end{array}$} & \multicolumn{2}{|c|}{ Kortste duur } & \multirow{2}{*}{$\begin{array}{c}\text { K.M } \\
\text { per } \\
\text { nur. }\end{array}$} \\
\hline & & & & min. & $\begin{array}{l}\text { Aantal } \\
\text { troinen. }\end{array}$ & & & $\min$. & $\begin{array}{l}\text { Aantal } \\
\text { troisen. }\end{array}$ & \\
\hline $1 b$ & Amsterd.-Haarlem-den Haag & 63 & 8 & 51 & 6 & 74. & 11 & 51 & 3 & 74 \\
\hline 10 & -den Haag & $4 \overline{5}$ & - & - & - & - & 4 & 37 & 3 & 73 \\
\hline $1 d$ & Arnsterdam-Leiden & 48 & 1 & 40 & - & 72 & - & - & - & \\
\hline $2 a$ & Amsterdam-Gouda & 52 & 1 & 47 & - & 67 & 3 & 44 & - & 71 \\
\hline $2 b$ & Nieuwersluis-den Haag. & 61 & 1 & 55 & - & 66 & $=$ & - & - & \\
\hline $2 c$ & Nienwersluis-Rotterd. Mars & 53 & 1 & 45 & - & 71 & - & - & - & - \\
\hline \multirow[t]{2}{*}{$2 d$} & Amsterdam-Gouda-Rotter- & & & & & & & & & \\
\hline & $d a$ & 73 & - & - & - & - & - & - & - & Co \\
\hline $\begin{aligned} 2 e I \\
2 e I I\end{aligned}$ & Rotterdam Maas-Utrecht & 5 & - & 一 & 一 & - & & 46 & 1 & 69 \\
\hline $2 f$ & $\begin{array}{l}\text { Rotterdam D.P.-Utrecht. } \\
\text { Den Haag-Utrecht. }\end{array}$ & 58 & 二 & - & - & - & - & - & - & - \\
\hline $2 g$ & Utrecht-Arnhe & .56 & 8 & 53 & 1. & 64 & 13 & 54 & 1 & $\overline{62}$ \\
\hline $2 h$ & Utrecht-Ede & 41 & 1 & 42 & - & 59 & 2 & 40 & 1 & 62 \\
\hline 2 & gen-Arnhem & 45 & 2 & 46 . & 1 & 59 & 5 & 44 & 1 & 61 \\
\hline 3 & Zwolle. & 77. & - & & - & & - & & - & \\
\hline $4 a$ & -Anersfoort & .68 & 4 & 60 & 2 & 68 & 10 & 59 & 1 & 69 \\
\hline $4 b$ & -Utrech $t$ & 89 & - & - & - & - & - & - & - & \\
\hline 5 & -Zutphen & 45 & - & -- & - & - & - & - & - & \\
\hline $6 a$ & ldoorn & 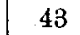 & 4 & 38 & 1 & 68 & 9 & 36 & 1 & 72 \\
\hline $6 b$ & sfoort. & 4 & 3 & 40 & 1 & 69 & 8 & 37 & 1 & 75 \\
\hline $6 c$ & t. & 97 & - & 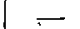 & - & - & - & - & - & \\
\hline Gid & -Apeld & 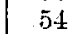 & - & - & 一 & _- & - & - & _- & \\
\hline $6 e$ & $-\mathrm{A}$ & 112 & - & - & - & - & - & 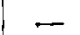 & - & \\
\hline $7 a$ & -den Bosch & 48 & 8 & 45 & 1 & 64 & 6 & 48 & 2 & 60 \\
\hline $7 b$ & aal-den Bosch & 6 & 2 & 55 & $\hat{I}$ & 75 & - & $x$ & - & \\
\hline $8 a$ & rt—Vork (Elst) & 5 & 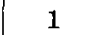 & 44 & 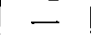 & 68 & 2 & 40 & 2 & 75 \\
\hline $8 b$ & $\mathrm{D}$ & 0 & $1^{*}$ & 7 & - & 68 & $1^{4}$ & 88 & - & 68 \\
\hline $8 c$ & (Elst) & 9 & $1^{*}$ & 85 & - & 6 & $1^{*}$ & 78 & - & 71 \\
\hline $8 d$ & $\mathrm{~N}$ & 0 & $2^{*}$ & 57 & 1 & 64 & - & & — & \\
\hline 9o & ndaal & 4 & 4 & 59 & 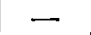 & 50 & 2 & 58 & 1 & 51 \\
\hline $9 b \mathrm{I}$ & -Esschen & 5 & - & - & $\ldots$ & - & - & - & 1 & \\
\hline $9 b \perp I$ & -Esschen & & - & - & . & -_ & 1 & 62 & - & 33 \\
\hline $0 a$ & $-B o$ & 138 & 1 & 110 & - & 75 & 1 & 113 & - & 5 \\
\hline & sendaal & & $5^{*}$ & 61 & $1^{*}$ & 7 & 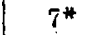 & 60 & 1 & 5 \\
\hline $10 e$ & $\mathrm{l}-\mathrm{B}$ & 6 & 4 & 2 & $1^{*}$ & 7 & $\dot{4}$ & 51 & $1 *$ & 74 \\
\hline $10 f$ & -Tilburg & \pm & $3^{4}$ & 36 & & 77 & 1 & 37 & 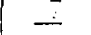 & 75 \\
\hline 11 & Bor & 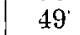 & $6^{*}$ & 45 & $1 *$ & 65 & $7^{*}$ & 43 & $1^{*}$ & 69 \\
\hline 12 & n-Weert-Roermond & 53 & - & - & 一 & $\therefore$ & 一 & - & - & 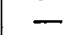 \\
\hline 1 & arden . . & 5 & - & - & -1 & - & - & - & - & - \\
\hline \multirow[t]{2}{*}{14} & Amsterdam-Hoorn. & 43 & - & - & - & - & - & $\dot{-}$ & - & - \\
\hline & 0 & & 72 & & & & 02 & & & \\
\hline
\end{tabular}

Op de met * gemerkle trajecten ontbrak de $3^{\circ}$ klasse. 
zonder oponthoud afgelegd.

\begin{tabular}{|c|c|c|c|c|c|c|c|c|c|c|c|}
\hline \multicolumn{4}{|c|}{1914} & \multicolumn{4}{|c|}{1916} & \multicolumn{4}{|c|}{1918} \\
\hline \multirow{2}{*}{$\begin{array}{l}\text { Aantal } \\
\text { treinen. }\end{array}$} & \multicolumn{2}{|c|}{ Kortste duur } & \multirow{2}{*}{$\begin{array}{c}\text { K.M. } \\
\text { per } \\
\text { uur. }\end{array}$} & \multirow{2}{*}{$\begin{array}{l}\text { Aantal } \\
\text { treinen }\end{array}$} & \multicolumn{2}{|c|}{ Kortste duur } & \multirow{2}{*}{$\begin{array}{l}\text { K.M. } \\
\text { per } \\
\text { uur. }\end{array}$} & \multirow{2}{*}{$\begin{array}{l}\text { Aantal } \\
\text { treinen. }\end{array}$} & \multicolumn{2}{|c|}{ Kortste duur } & \multirow{2}{*}{$\begin{array}{c}\text { K.M. } \\
\text { per } \\
\text { uur. }\end{array}$} \\
\hline & $\min$. & $\begin{array}{c}\text { Aantal } \\
\text { treinen. }\end{array}$ & & & $\min$. & $\begin{array}{c}\text { Aantal } \\
\text { troinen. }\end{array}$ & & & $\min$. & $\begin{array}{c}\text { Aantal } \\
\text { troinen. }\end{array}$ & \\
\hline 21 & 48 & 13 & 79 & 19 & 48 & 13 & 72 & 2 & 58 & 1 & 65 \\
\hline 5 & 35 & 3 & 77 & 6 & 35 & 3 & 77 & (Zo.) & 45 & - & 60 \\
\hline- & - & - & - & - & $\vec{a}$ & - & - & - & - & - & - \\
\hline 2 & 48 & 1 & 65 & 6 & 51 & - & 61 & - & 一 & - & - \\
\hline - & - & - & - & - & - & - & - & - & $\div$ & - & - \\
\hline- & - & - & - & - & - & $\longrightarrow$ & $\dot{-}$ & $\rightarrow$ & - & - & - \\
\hline 6 & 55 & - & 81 & - & 一 & - & - & - & - & - & - \\
\hline 6 & 43 & 1 & 81 & 1 & 57 & - & 56 & - & - & - & - \\
\hline 5 & 54 & $\mathrm{I}$ & 64 & 6 & 54 & 1 & 68 & - & - & - & - \\
\hline- & - & - & - & 3 & 54 & 1 & 64 & - & - & - & - \\
\hline 19 & 50 & 12 & 67 & 11 & 50 & 9 & 67 & - & - & - & $\ldots$ \\
\hline 4 & 33 & 1 & $74 t$ & 1 & 36 & + & 68 & - & - & - & - \\
\hline$\rightarrow$ & - & - & - & 1 & 39 & - & 69 & - & - & - & - \\
\hline 3 & 64 & 1 & 72 & 3 & 67 & 1 & 69 & - & - & - & - \\
\hline 8 & 55 & 5 & 73 & 11 & 55 & 5 & 73 & 6 & 70 & - & 58 \\
\hline 6 & 75 & 5 & 71 & - & 一 & - & $\longrightarrow$ & - & - & - & - \\
\hline 6 & 40 & 3 & $67 t$ & - & - & ㄴ & - & - & - & - & - \\
\hline 9 & 35 & 2 & $74^{2}$ & 10 & 36 & - & 72 & - & - & 一 & - \\
\hline 7 & 39 & 5 & 71 & $\check{\mathbf{u}}$ & 39 & 3 & 71 & $\dot{-}$ & - & - & - \\
\hline 4 & 78 & 1 & $74 \frac{1}{2}$ & - & - & - & - & - & - & - & - \\
\hline 1 & 46 & - & $70^{2}$ & $\cdots$ & 一 & 一 & - & - & - & - & - \\
\hline- & - & 一 & - & 1 & 93 & - & 72 & - & - & 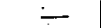 & $\cdot-$ \\
\hline 10 & 45 & 1 & 64 & 8 & 45 & 1 & 69 & 2 & 54 & 1 & 53 \\
\hline- & - & - & - & $\ldots$ & - & - & - & - & - & - & - \\
\hline 3 & 40 & 1 & 75 & 1 & 40 & - & 75 & - & - & 一 & - \\
\hline $1^{*}$ & 75 & 一 & 79 & - & - & - & - & - & - & - & - \\
\hline 2 & 70 & $1^{*}$ & 79 & 1 & 73 & - & 71 & - & - & - & - \\
\hline 一 & - & - & - & - & 一 & - & $\longrightarrow$ & - & - & - & - \\
\hline 2 & 58 & - & 51 & - & - & - & - & - & - & - & - \\
\hline 2 & 64 & - & $52 \frac{1}{2}$ & - & - & - & - & - & - & - & - \\
\hline 1 & 60 & - & $55^{2}$ & - & 一 & - & - & - & $\longrightarrow$ & - & - \\
\hline 2 & 110 & - & 75 & - & - & - & - & - & - & - & - \\
\hline 10 & 60 & 4 & 75 & 1 & 65 & - & 69 & - & - & - & - \\
\hline 6 & 50 & 1 & $75 \frac{1}{2}$ & - & - & - & - & - & - & - & - \\
\hline- & - & 一 & - & - & - & - & 一 & - & - & - & - \\
\hline 9 & 42 & 3 & .70 & - & - & - & - & - & -- & - & - \\
\hline 6 & 50 & - & $63 \frac{1}{2}$. & 6 & 50 & 4 & $63 \frac{1}{2}$ & 4 & 53 & $=$ & 60 \\
\hline 3 & 48 & 1 & $69^{2}$ & 1 & 55 & - & 60 & - & - & 二 & - \\
\hline - & - & - & - & 2 & 40 & 1 & 65 & - & - & - & - \\
\hline 169 & & & & 110 & & & & $\left.14^{\prime}\right)$ & & & \\
\hline
\end{tabular}

1) en 1 op Zondag. 